\title{
Reframing family-centred obesity prevention using the Family Ecological Model
}

\author{
Kirsten K Davison ${ }^{1, *}$, Janine M Jurkowski ${ }^{2}$ and Hal A Lawson ${ }^{3}$ \\ ${ }^{1}$ Department of Nutrition, Harvard School of Public Health, 665 Huntington Avenue, Boston, MA 02115, USA: \\ ${ }^{2}$ Department of Health Policy, Management and Behavior, School of Public Health, University of Albany, \\ Rensselaer, NY, USA: ${ }^{3}$ Department of Educational Administration \& Policy Studies and School of Social Welfare, \\ University of Albany, Albany, NY, USA
}

Submitted 6 March 2012: Final revision received 27 June 2012: Accepted 12 July 2012: First published online 22 0ctober 2012

\begin{abstract}
Objective: According to the Family Ecological Model (FEM), parenting behaviours are shaped by the contexts in which families are embedded. In the present study, we utilize the FEM to guide a mixed-methods community assessment and summarize the results. Additionally, we discuss the utility of the FEM and outline possible improvements.

Design: Using a cross-sectional design, qualitative and quantitative methods were used to examine the ecologies of parents' cognitions and behaviours specific to children's diet, physical activity and screen-based behaviours. Results were mapped onto constructs outlined in the FEM.

Setting: The study took place in five Head Start centres in a small north-eastern city. The community assessment was part of a larger study to develop and evaluate a family-centred obesity prevention programme for low-income families. Subjects: Participants included eighty-nine low-income parents/caregivers of children enrolled in Head Start.

Results: Parents reported a broad range of factors affecting their parenting cognitions and behaviours. Intrafamilial factors included educational and cultural backgrounds, family size and a lack of social support from partners. Organizational factors included staff stability at key organizations, a lack of service integration and differing school routines. Community factors included social connectedness to neighbours/friends, shared norms around parenting and the availability of safe public housing and play spaces. Policy- and media-related factors included requirements of public assistance programmes, back-to-work policies and children's exposure to food advertisements.

Conclusions: Based on these findings, the FEM was refined to create an evidencebased, temporally structured logic model to support and guide family-centred research in childhood obesity prevention.

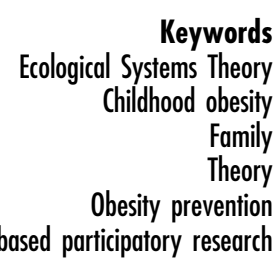

Childhood obesity is a national and international public health problem ${ }^{(1,2)}$ with detrimental effects on children's health and educational outcomes ${ }^{(3)}$. Parents and caregivers play a central role in shaping children's lifestyle behaviours $^{(4,5)}$ as evidenced by research linking parents' attitudes, beliefs and parenting strategies with children's dietary intake, physical activity and sedentary behaviours $^{(6-8)}$. Accordingly, engaging parents/caregivers in childhood obesity prevention is a recommended strategy to allay the epidemic of childhood obesity ${ }^{(9)}$.

Unfortunately, parents and families currently are not featured in public health discourse on childhood obesity prevention. Recent policy and practice reports focus on the availability of sugar-sweetened beverages in public settings, food marketing standards, child care and school policies, the role of health-care providers and agricultural policy $^{(10)}$. Families do not feature as a centre-point for research or policy development. The 2011 Cochrane review of childhood obesity prevention programmes provides further evidence of this pattern; just fourteen out of fifty-five programmes included a family component ${ }^{(11)}$. In most cases, a family component was an 'add on' to a larger school-based programme and parents were typically engaged using passive methods such as newsletters and family fun nights. Not surprisingly, this approach has failed to engage parents ${ }^{(12,13)}$ and improve children's obesity-related outcomes $^{(14)}$.

Today's research agendas and policy reports emphasize the crucial role of contextual factors in health behaviour and outcomes. This focus has been heavily shaped by 
Ecological Systems Theory (EST). EST states that human behaviour cannot be understood without taking into consideration the contexts in which it occurs ${ }^{(15-17)}$. Over the past two decades, EST and other contextual theories have been instrumental in the development of public health programmes that focus less on individual beliefs, attitudes and knowledge and more on environments that shape children's behaviour such as schools and communities.

Unfortunately, EST has not been widely utilized to design family interventions for obesity prevention or to raise the profile of families in public health discourse on childhood obesity prevention. To effectively design and implement family-centred interventions, especially culturally responsive interventions, it is imperative to understand the broader context in which parenting takes place. In combination with cultural factors, these ecological and family system factors may help explain parenting cognitions and behaviours that promote or discourage healthy lifestyles in children.

The Family Ecological Model (FEM), illustrated in Fig. 1, was developed to account for contextual and family systems factors affecting parenting specific to healthy lifestyles. This model mimics EST, but emphasizes the family - rather than the individual - as the focal point of the model and the intervention target. The inner circle of the FEM summarizes the processes by which parents influence children's diet-, activity- and screenbased behaviours, including parents' knowledge and beliefs about obesity, modelling of healthy behaviours and opportunities they create for healthy eating and physical activity. Research documents the important role of each of these factors in predicting children's lifestyle behaviours $^{(18)}$. The model's outer domains represent theoretically justifiable, contextual factors derived from EST including demographic factors, child characteristics, organizational characteristics, community characteristics, and media and policy factors.

While this model is heuristically useful, it has not been empirically tested. Recently, we addressed this gap. We utilized the FEM to structure a mixed-methods community assessment which served as formative work for the development of a family-centred childhood obesity prevention programme targeting low-income families. This application, and the resulting data, provided the opportunity to test and refine the FEM, to strengthen the links between theory and application in real-world contexts ${ }^{(18)}$ and provide a tool to bring families into public health discourse on childhood obesity prevention. Herein, we: (i) summarize results from the community assessment; (ii) examine the extent to which the data compiled support the contextual components of the FEM (i.e. the validity of the FEM); (iii) assess the FEM's utility in generating ideas for the development of a family-centred intervention; and (iv) outline an empirically grounded revision of the FEM for future research, intervention and public health reports.

\section{Methods}

\section{Research setting}

The present study is nested within a 2-year study funded by the National Institutes of Health to develop, implement and evaluate a family-centred childhood obesity prevention programme for low-income families

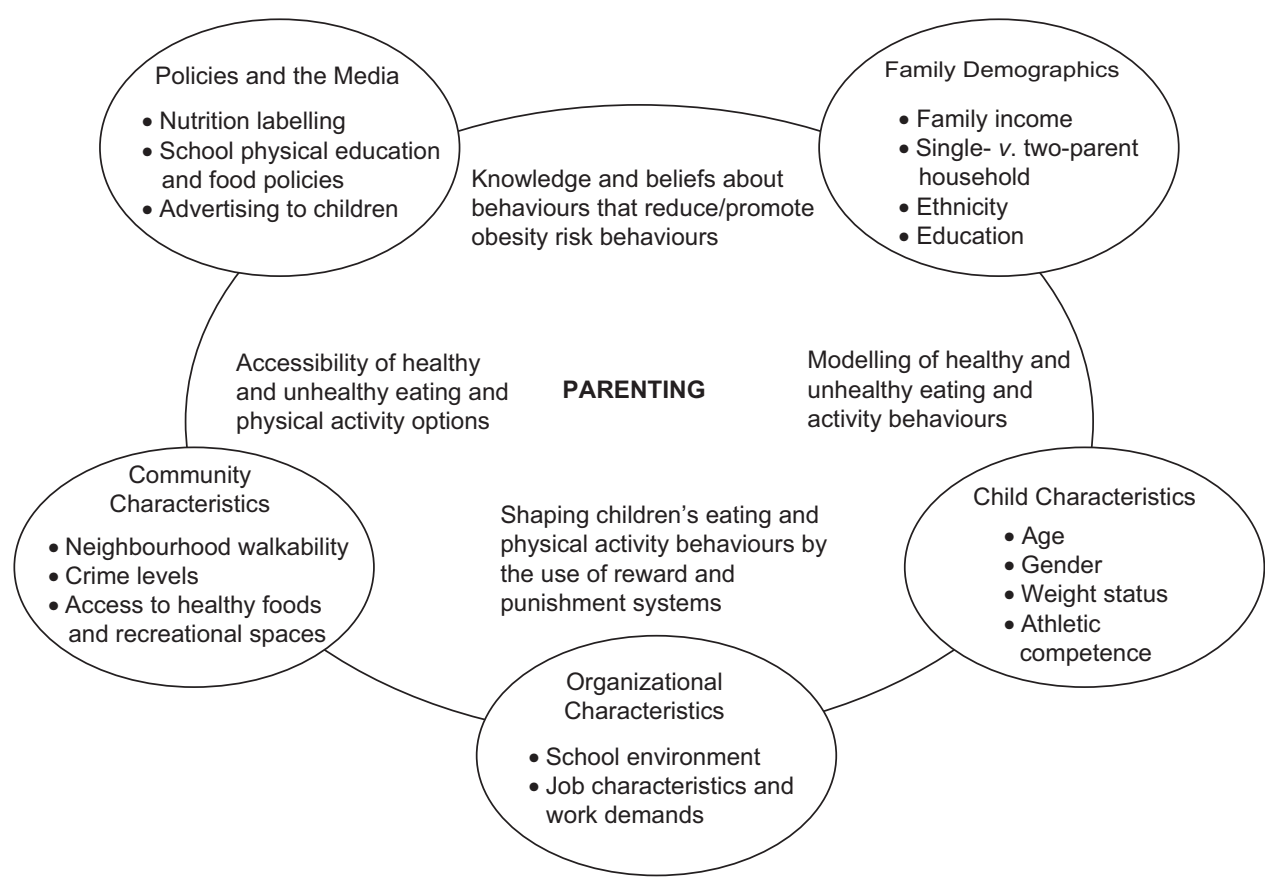

Fig. 1 The original Family Ecological Model ${ }^{(18)}$ 
with children of pre-school age. It focuses on formative work during the first year of the study which included a comprehensive community assessment. The study took place in five Head Start centres, serving over 400 children and their families, in a small, medically underserved, north-eastern city.

\section{Procedure}

The study utilized community-based participatory research (CBPR). According to Viswanathan et al. ${ }^{(19)}$ (p. 3), CBPR is 'a collaborative research approach that is designed to ensure and establish structures for participation by communities affected by the issue being studied, representatives of organizations, and researchers in all aspects of the research process to improve health and well-being through taking action, including social change'. Our CBPR approach, which we refer to as 'family-centred CBPR', was unique because parents of Head Start children served as co-researchers and had equal decision-making power in the research process.

A community advisory board (CAB) consisting predominantly of parents of children enrolled in Head Start centres was established. Additional members of the board included representatives from local community organizations, a large paediatric provider and a local church for a total of eighteen members. On average, CAB meetings were held monthly. One of the CAB's first objectives was to develop an extensive community assessment to learn more about the daily realities of families, programme outcomes valued by Head Start families, and family and community resources that could be utilized to foster sustainability.

The FEM was used to frame CAB members' discussions around possible constructs to include in the community assessment. CBPR leaders provided CAB members with a copy of the FEM and explained the components and purpose of the model. As parents discussed factors in their social, cultural, economic and physical environments that affected their food, physical activity and screen-based parenting practices, CBPR leaders encouraged them to consider the relevance of each contextual factor outlined in the model. One outcome of this process was the creation of an extensive list of factors that parents believed affected their parenting cognitions and behaviours (see Table 1). These factors were subsequently measured in the community assessment using a combination of qualitative and quantitative methods.

The community assessment was conducted from February to May 2010. Families were recruited through posters displayed in Head Start centres and flyers sent home with children. Recruitment was conducted separately for each method of assessment. While there was some overlap in participants across methods, this was not a recruitment goal. The study was conducted according to the guidelines in the Declaration of Helsinki and all procedures involving human subjects were reviewed and approved by the Internal Review Board and the Office for Research Compliance at the University of Albany. All participants signed a written informed consent and were compensated for their involvement with gift cards ranging in value from \$US 10 to \$US 20. Additionally, parents serving as $\mathrm{CAB}$ members were compensated with a \$US 25 gift card for each board meeting attended.

\section{Measures}

Survey

A 25-item survey was used to gather information about food, physical activity and screen-based parenting, the roles of adults and older children in the household, family

Table 1 Constructs examined during the community assessment by dimension of the Family Ecological Model and method of assessment

\begin{tabular}{ll}
\hline Constructs assessed in the community assessment & Method of assessment \\
\hline Family demographics* & Survey \\
Other adults in the home who help care for the child & Focus groups \\
Family economic challenges and implications for parenting & Survey \\
Family health concerns (e.g. diabetes) & Photovoice, focus group \\
Relationship between the child's mother and father and differences in parenting strategies & Interview \\
Daily stress in the family and sources of stress for parents (e.g. meal preparation) & Focus groups \\
Family cultural beliefs around food, physical activity and obesity & Focus groups \\
Parents' self-efficacy to create healthy lifestyles for themselves and their children & Survey, focus groups \\
Child characteristics and sibling characteristicst & Survey, focus groups \\
Number, age and age range of children in the household & Focus groups \\
Role of older siblings in the care of younger children & Focus groups \\
Managing conflict between siblings & Interview \\
Organizational factors affecting parenting & Interview \\
Head Start policies (e.g. closing time, days closed) & Interview \\
Head Start resources to assist families with an overweight child & Survey \\
Family engagement in institutional activities (e.g. Head Start family events) &
\end{tabular}

*Interactions with the advisory board indicated that the term 'family demographics' was too narrow and needed to be expanded to include additional components of the family environment including interactions between parents, the general stress level in families.

tSibling characteristics were not included in the original formulation of the Family Ecological Model but emerged through discussions with board members as an important child-specific factor influencing parenting. 
utilization of community-based programmes and services, and parents' viewpoints on healthy lifestyles and meanings assigned to childhood overweight. The survey included a combination of closed- and open-ended response formats. Parents completing the survey were also invited to complete a brief follow-up interview which expanded on these questions.

\section{Focus groups}

Three focus groups with twenty-seven parents, five to twelve parents per group, examined the impact of having children across a wide age range on parenting specific to obesity, which was identified as a pertinent issue during discussions with the advisory board members. Recruitment for the focus groups focused on Head Start parents who also had a child who was $5+$ years older than the Head Start child.

\section{Photovoice}

Photovoice methodology ${ }^{(20)}$ was used to supplement the information gathered through the focus groups. Twelve parents were given disposable cameras and asked to document through photographs what typically caused stress in their home and community. They were asked to document factors that made it difficult (or easy) to take care of their family. In a follow-up discussion, parents were given printed copies of their photographs and asked to select the five most salient pictures to share with the group and explain their rationale for taking each picture. The focus groups and Photovoice discussion were audio recorded and transcribed.

\section{Windshield survey}

Six parents completed a windshield survey. This assessment asks respondents to talk while driving (or being driven) through the neighbourhood ${ }^{(21)}$. Each parent led a driving tour of their neighbourhood and responded to structured open-ended questions about their neighbourhood based on an interview guide. The interview guide was developed to capture perceived social, economic and environmental conditions of the neighbourhood in relation to their daily activities, parenting and their children's well-being. Parents' responses were audio recorded and photographs of key environmental factors were taken.

\section{Data analysis and model review}

Analysis of the qualitative data was conducted by a member of the research team and a parent from the CAB. Both individuals read the transcripts and coded the text based on the larger themes of the FEM. They discussed the quotations and themes and generated pattern codes to discern relationships between situations and experiences with parenting beliefs and practices ${ }^{(22)}$.

Parents' survey responses were entered into a data-entry program and descriptive statistics were generated for each question. Open-ended responses from the follow-up interview were entered verbatim into a Microsoft ${ }^{\circledR}$ Excel file. Responses were reviewed and sorted around the themes identified. Following coding and analysis of the data for each methodology, the results were compiled across methodologies to provide summaries for each of the contextual dimensions of the FEM.

Results from the community assessment were reviewed with the $\mathrm{CAB}$ through a series of data workshops, which enabled parents to provide their interpretation of the data. Results were also shared with Head Start families (beyond the advisory board) at a Town Hall meeting and with Head Start staff and management and the broader community through a community forum to solicit further input on the interpretation of the data and the themes emerging. The data and interpretative feedback were used to refine the FEM and identify priorities for the subsequent family-centred intervention.

\section{Results}

\section{Participant characteristics}

Participants included a total of eighty-four parents (or caregivers), of whom 91\% were female. Additional information on participant characteristics is presented in Table 2.

\section{Family ecologies}

\section{Family demographics}

Intrafamilial issues, referred to as Family Demographics in the original FEM, included the availability of other adults in the home to assist with child care and parents' perceptions of economic factors affecting parent decision making, family health concerns, stressors of daily living, family and cultural beliefs, family interpersonal dynamics and parent mental health.

Table 2 Participant characteristics: low-income parents/caregivers of pre-school children enrolled in five Head Start centres in a small north-eastern US city, February to May 2010

\begin{tabular}{lc}
\hline Characteristic & $n$ or \% \\
\hline Total number of parents/caregivers & 89 \\
Caregiver gender (\% female) & 91 \\
Number of children living in the home & $2 \cdot 61$ \\
Mean & $1 \cdot 38$ \\
SD & \\
Race/ethnicity (\%) & 52 \\
Non-Hispanic white & 22 \\
Black or African American & 6 \\
Hispanic black & 10 \\
Hispanic white (or not specified) & 10 \\
Unknown or not reported & \\
Number of participants per procedure & 7 \\
Windshield survey & 25 \\
Focus groups & 8 \\
Photovoice & 57 \\
Survey & 38 \\
Follow-up interview & \\
Number of procedures completed (\% of parents) & 92 \\
1 & 8 \\
2 & \\
\hline
\end{tabular}


Although the majority of participants were single parents, $53 \%$ reported the presence of another adult in the home who assisted with child care and 19\% reported that an older child provided care for younger children; $17 \%$ reported both. When discussing economic factors that affected parenting, parents provided explicit examples of knowing what was 'right' but not being able to follow through due to financial limitations. For example, parents expressed a strong desire to enrol their children, particularly boys, in sports but withdrew them because they could not afford the costs of participation. Parents believed they were knowledgeable about the right foods to feed their children but their actual choices were constrained by time and the costs of healthy food.

The daily experience of caring for young children, parents' mental health and parents' life histories and cultural beliefs also had implications for parenting. A common theme among parents was the lack of time they had for themselves. A number of parents expressed disappointment with their own lives. Parents felt that they had given up their own needs and interests to be the sole provider for their children.

Intergenerational family patterns regarding food preferences and eating habits also emerged. Parents discussed how they tended to cook for their children the foods that they themselves liked and their parents had cooked for them. Other parents discussed not knowing how to cook because they were never shown. Conversely, one parent discussed at great length the emphasis placed on healthy eating in her family which resulted from a family member needing to manage diabetes.

Parents also discussed the effects of their life histories on their parenting. Specifically, parents, many of whom had been teen mothers, discussed how their parenting had changed and evolved as they themselves had matured.

Cultural beliefs emerged as important factors. There was a general consensus among parents that it is good for a child to be slightly overweight because it shows that they can afford to feed their child. Having a skinny child threatened their aspiration to be viewed as good, competent parents. In parents' views, a skinny child was associated with an inaccurate social signal about their identities (bad parent) and lifestyles (e.g. drug use or HIV status). Overall, parents believed that if a child was active then s/he was the right weight regardless of body size.

\section{Child characteristics}

Parents emphasized child and family characteristics that influenced their parenting cognitions and behaviours. Characteristics included the number of children in the family, the age range of children, the presence of older siblings and having a child with special needs. Approximately $50 \%$ of families had two children living in the home, $25 \%$ had three children and $25 \%$ had four or more children. Many families had children across a broad age range; $30 \%$ of parents had a child of pre-school age and one or more children aged 11 years or older.
Parents reported benefits and challenges associated with having children of diverse ages. Benefits included older boys 'filling in' for an absent father by looking out for younger children and older girls assisting with meal preparation. Older siblings were helpful in supervising younger children. Associated challenges that parents encountered included older children instigating bad behaviour in younger children and the difficulty of meeting the diverse needs of their children while maintaining a job. Sibling conflict around selecting television programmes was mentioned repeatedly by parents. One solution was for each child to have a television in his/her room. Finally, given that a special health care need is one criterion for entry into Head Start, many families had a child with a disability which brought the added stress of coordinating multiple medical and specialist appointments.

\section{Organizational characteristics}

Parents' accounts of organizational factors affecting parenting behaviours generally focused on Head Start staff and policies. Parents generally reported positive, supportive relationships with their child's teacher and their family advocate. The degree to which parents communicated with Head Start staff and reached out to them for support and advice, however, varied greatly across parents. The generally positive relationships with teachers highlight the potential to work with pre-school teachers when trying to reach parents of young children.

Parents were generally aware of, and saw benefit in, the resources provided by Head Start (e.g. letters sent home with their children's health screening results). Some Head Start policies, however, created challenges for parents. For example, centre closing times, which ranged between $14 \cdot 30$ and $17 \cdot 00$ hours, were not conducive to working parents. Such policies created challenges for parents managing multiple children, especially ones trying to maintain employment.

\section{Neighbourbood/community characteristics}

Parents identified 'neighbourhood effects' on their family ecologies. Key topics included a lack of trust of people in the neighbourhood, housing instability, a lack of safe play spaces and supermarkets, low performing schools, poor access to public transportation and poor relationships with health-care providers.

Parents discussed concerns about their children socializing with other children or being around older children from the neighbourhood. As one parent noted 'their mouths are reckless and my daughter don't talk like that'. Some parents only allowed their children to play outside under tight supervision and did not let their children walk to school. Parents expressed grave concern about sexual predators. They were knowledgeable of registered sex offenders in their neighbourhoods and described the cars and hang-out locations of specific ones near their homes. Overall, parents felt disconnected from people living 
around them and a need to prevent their children from interacting with non-family members.

Many parents were challenged by chronic stress. Some of this stress was associated with pervasive instability. Housing insufficiency and instability were a recurrent challenge. Families frequently had to move residences, often with last-minute notice. Sometimes they moved for reasons outside their immediate control (e.g. due to code violations of the rental building or the rental property going into foreclosure). Mothers generally had to deal with such moves alone and with little access to resources to facilitate moving.

Parents with more than one child had an additional situational challenge. They often had children who attended schools in different towns or counties. In addition to having children over a broad age range, this pattern stemmed in part from parents' efforts to ensure that their children did not attend schools that were of poor quality and/or unsafe. Parents reported driving long distances at variable times of the day, due to differences in school start and end times. These transportation challenges taxed parents' time, thus making it difficult for them to maintain a job and to attend to domestic duties such as cooking meals. Moreover, younger children often spent long periods of time in the car.

Results from the windshield surveys indicated that there were few safe outdoor play areas in neighbourhoods. Parks and playgrounds were often run down, with minimal equipment, and were considered a haven for adolescents and drug dealers. As a result, families tended not to use them. Additionally, there were no major grocers in most neighbourhoods. These resource gaps, coupled with poor public transportation infrastructure (which is common in small cities) and a reliance on food stamps, had a direct impact on meal planning. For example, food stamps limited their food choices; parents had to purchase the majority of foods once a month and some parents transported their groceries on buses. These factors influenced what they bought in terms of food that lasts a month and products that they could carry.

Finally, parents reported a lack of access to nearby, quality health-care services. They discussed difficulties in obtaining mental health services, finding providers who accepted Medicaid and accessing specialized services for children with special needs. Likewise, many parents reported a sense of distrust of their providers and felt that the quality of providers accepting Medicaid was lower.

\section{Policy and the media}

The final ecological context reviewed focused on the macro-level factors of media and policy. Parents reported a wide range of sources from which they acquired information about healthy living. The most commonly reported source of health information was the doctor, with over $80 \%$ of parents relying on this source. Additionally, $40 \%$ of parents relied on previous life experiences or information received through WIC (Special Supplemental Nutrition Program for Women, Infants, and Children). A heavy reliance on doctors for health information presents a quandary for parents given their negative perception of medical providers in their area.

A number of policies and public assistance requirements had a direct effect on the family environment. Parents experienced challenges finding affordable housing and felt pigeon-holed in unsafe neighbourhoods with known sexual predators. This in turn affected the extent to which families ventured outdoors. Similarly, due to distrust of the Department of Social Services, parents reported a reluctance to answer their door to people who looked professional. These factors, combined with their financial situation, resulted in families not utilizing services available in their communities and having a limited ability to create a network of trusted people (other than family members) for social support.

\section{Discussion}

Findings from the present study support the validity of the FEM; constructs outlined in the FEM were identified in the data. Moreover, consistent with the FEM, findings illustrate the complexity of family life, particularly for lowincome families, and highlight the futility of interventions that emphasize parent education without careful consideration for the context in which parenting takes place. Beyond the FEM, the study identified a number of assets in families that could be leveraged in preventive interventions, including older siblings and other adults in the home who could assist with younger children and positive relationships between parents and their children's teachers. Additional assets included parents' willingness to trust other parents, their awareness of their growth as parents and their knowledge of dangers in their communities.

While these findings support the validity of the FEM, they revealed a number of weaknesses in its utility. First, the term 'family demographics' (as labelled in the original FEM) was clearly too narrow to reflect the breadth of intrafamilial factors identified. Constructs such as parenting efficacy, family and cultural beliefs and the realities of daily life cannot be readily subsumed under this title. Second, while the FEM provided much needed guidance in structuring the community assessment and resulted in a wealth of information about families and family life, it was less helpful in structuring the generation of ideas for the intervention and its evaluation. Here, the element of time, and hence potential mediators of programme effect, was missing.

The FEM was revised to address these shortcomings (see Fig. 2; new elements are indicated in bold). The revised model takes the form of a logic model, illustrating an anticipated causal sequence, and is informed by theory, data from the present study and prior research examining parenting and children's health behaviours. As such, the 


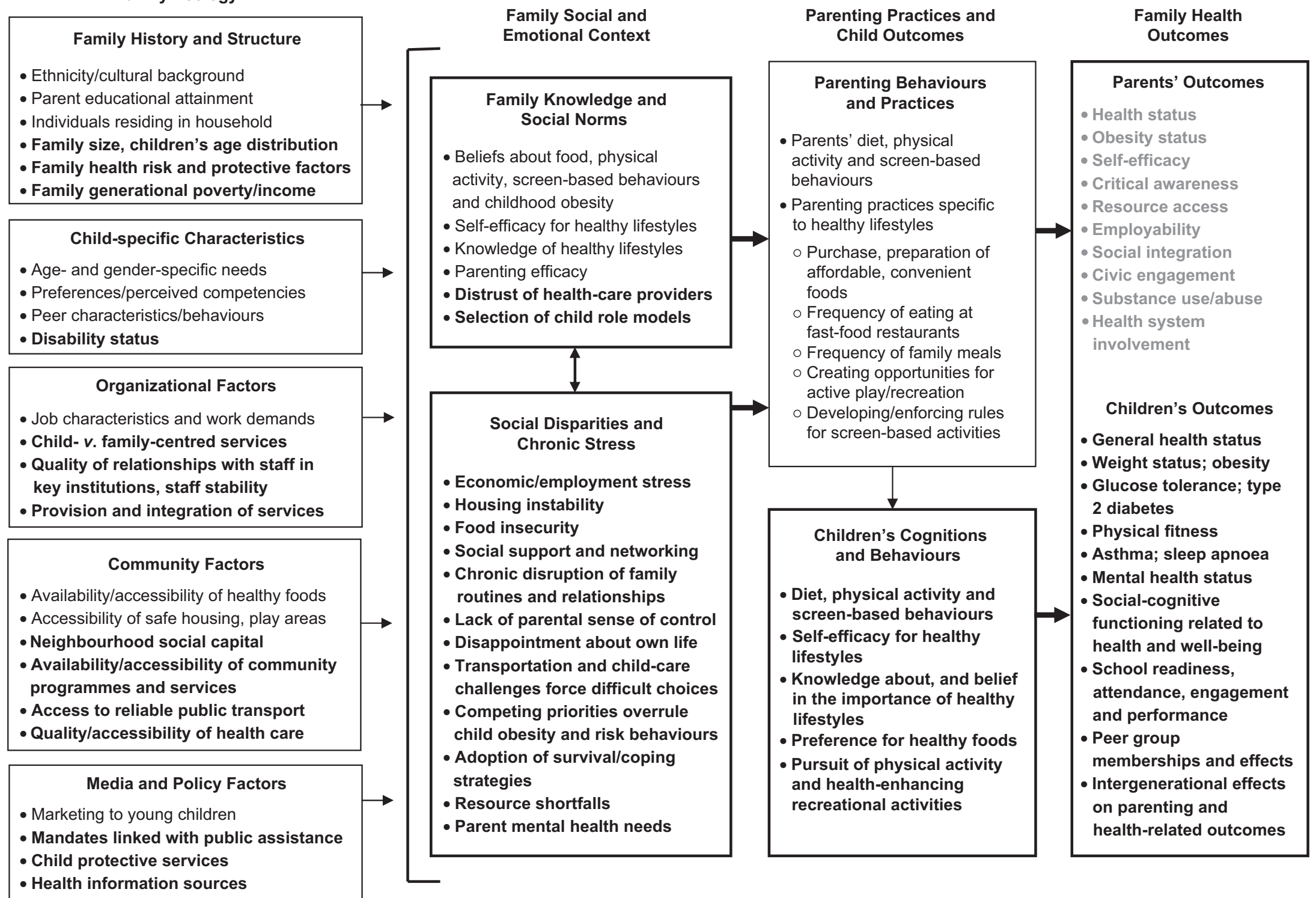

Fig. 2 The revised Family Ecological Model; bolded text and boxes indicate new components and constructs that were not part of the original model 
revised model reflects a blend of extant research and the findings reported herein. The fundamental components of the original FEM are listed in the far left column as the Family Ecology. The Social and Emotional Context of the family, resulting from the family ecology, reflects Family Knowledge and Social Norms as well as Social Disparities and Chronic Stress; we purposely selected the term 'social disparities' to emphasize that these characteristics or factors are socially constructed.

The social and emotional context of the family in turn shapes Parenting Behaviours and Practices (upper section of the third column) and Children's Cognitions and Behaviours (lower section of the third column). The parenting practices and child outcomes outlined are consistent with published research ${ }^{(7,23-26)}$. The final column includes research-supported long-term outcomes among children resulting from healthy parenting practices and healthy diet and physical activity behaviours ${ }^{(27-29)}$. In the absence of research, the outcomes for parents are hypothetical and are hence shown in a lighter shade.

The revised FEM is intended to communicate two testable hypotheses:

1. When components from the first two columns of the revised model are targeted (i.e. family ecological and social and emotional factors), family-centred interventions for childhood obesity are more likely to result in positive changes in parents' knowledge, cognitions and behaviour, resulting in improvements in their children's health.

2. When multiple components from the first column are addressed, positive programme effects are more likely to be sustainable.

Testing these hypotheses will require culturally competent interventions that prioritize parents and the family system, but also reach beyond them to modify the ecologies in which they are embedded. Both of these hypotheses, and the implied research, can be integrated into the current public health focus on ecological contexts.

Beyond the revised FEM, future research can benefit from the present study's novel use of family-centred $\mathrm{CBPR}^{(30)}$. Parents were co-researchers in the implementation, assessment and refinement of the FEM. They defined the scope of the assessment, assisted with data collection and participated in the interpretation of the qualitative data. Additional strengths include a focus on low-income families, who are at particular risk of obesity ${ }^{(31)}$, and the use of multiple methods to examine the family ecology. The study provides a rich and ecologically valid assessment of the FEM and a prototype for future research on families and childhood obesity.

\section{Acknowledgements}

Sources of funding: This research was supported by the National Center on Minority Health and Health Disparities,
National Institutes of Health (grant number R24MD001120) through the American Recovery and Reinvestment Act. This research was affiliated with the Center for the Elimination of Minority Health Disparities (grant number 1 P20 MD003373). The content is solely the responsibility of the authors and does not necessarily represent the official views of the National Center on Minority Health and Health Disparities or the National Institutes of Health. Conflicts of interest: None declared. Autbors' contributions: K.K.D. led the conception and design of the paper and the development of the revised FEM, co-led the acquisition of funding with J.M.J., participated in data collection, and drafted the manuscript. J.M.J. contributed to the conception and design of the paper and the revised model, co-led the acquisition of funding with K.K.D., led the collection and analysis of the qualitative data, and critically revised the manuscript for important intellectual content. H.A.L. contributed to the conception and design of the paper and the revised model, participated in the acquisition of funding with K.K.D. and J.M.J., and critically revised the manuscript for important intellectual content. All authors read and approved the final manuscript. Acknowledgements: The authors would like to thank the parents and grandparents of Communities for Healthy Living and the staff of the Commission on Economic Opportunities for the Greater Capital District.

\section{References}

1. Wang Y \& Lobstein T (2006) Worldwide trends in childhood obesity. Int J Pediatr Obes 1, 11-25.

2. Ogden C, Carroll M, Curtin L et al. (2010) Prevalence of high body mass index in US children and adolescents, 2007-2008. JAMA 3003, 242-249.

3. Daniels S (2006) The consequences of childhood overweight and obesity. Future Child 16, 47-67.

4. Davison K \& Birch L (2001) Childhood overweight: a contextual model and recommendations for future research. Obes Rev 2, 159-171.

5. Campbell K \& Hesketh K (2007) Strategies which aim to positively impact on weight, physical activity, diet and sedentary behaviours in children from zero to five years. A systematic review of the literature. Obes Rev 8, 327-338.

6. Birch LL \& Davison KK (2001) Family environmental factors influencing the developing behavioral controls of food intake and childhood overweight. Pediatr Clin North Am 48, 893-908.

7. Gustafson S \& Rhodes R (2006) Parental correlates of physical activity in children and early adolescents. Sports Med 36, 79-97.

8. Institute of Medicine (2005) Preventing Childhood Obesity: Health in the Balance. Washington, DC: The National Academies Press.

9. Dietz W \& Gortmaker S (2001) Preventing obesity and children and adolescents. Annu Rev Public Health 22, 337-353.

10. Institute of Medicine (2012) Accelerating Progress in Obesity Prevention: Solving the Weight of the Nation. Washington, DC: Institute of Medicine; available at http://www.iom.edu/Reports/2012/Accelerating-Progressin-Obesity-Prevention.aspx 
11. Waters E, de Silva-Sanigorski A, Hall BJ et al. (2011) Interventions for preventing obesity in children. Cochrane Database Syst Rev issue 12, CD001871.

12. Hingle MD, O'Connor TM, Dave JM et al. (2010) Parental involvement in interventions to improve child dietary intake: a systematic review. Prev Med 51, 103-111.

13. O'Connor TM, Watson K, Hughes S et al. (2010) Health professionals' and dietetics practitioners' perceived effectiveness of fruit and vegetable parenting practices across six countries. J Am Diet Assoc 110, 1065-1071.

14. Stice E, Shaw H \& Marti C (2006) A meta-analytic review of obesity prevention programs for children and adolescents: the skinny on interventions that work. Psychol Bull $\mathbf{1 3 2}$ 667-691.

15. Stokols D, Allen J \& Bellingham RL (1996) The social ecology of health promotion: implications for research and practice. Am J Health Promot 10, 247-251.

16. Bronfenbrenner U (1992) Ecological systems theory. In Six Theories of Child Development: Revised Formulations and Current Issues, pp. 187-249 [R Vasta, editor]. London: Jessica Kingsley Publishers.

17. Bronfenbrenner U \& Morris PA (1988) The ecology of human developmental processes. In The Handbook of Child Psychology, 3rd ed., pp. 993-1027 [W Damon and N Eisenberg, editors]. New York: John Wiley \& Sons.

18. Davison K \& Campbell K (2005) Opportunities to prevent obesity in children within families: an ecological approach. In Obesity Prevention and Public Health, pp. 207-230 [D Crawford and R Jeffery, editors]. Oxford: Oxford University Press.

19. Viswanathan M, Ammerman A, Eng E et al. (2004) Community-based Participatory Research: Assessing the Evidence. AHRQ Publication O4-E0221-1. Summary Evidence Report/Technology Assessment no. 99. Rockville, MD: Agency of Healthcare Research and Quality; available at http://www.ahrq.gov/downloads/pub/evidence/pdf/cbpr/ cbpr.pdf
20. Wang C \& Burris M (1997) Photovoice: concept, methodology, and use for participatory needs assessment. Health Educ Behav 24, 369-387.

21. Barnett E, Anderson T, Blosnich J et al. (2007) Heart Healthy and Stroke Free: A Social Environment Handbook. Atlanta, GA: Department of Health and Human Services, Centers for Disease Control and Prevention; available at http://www.cdc.gov/dhdsp/docs/seh_handbook.pdf

22. Miles M \& Huberman A (1994) Qualitative Data Analysis: An Expanded Sourcebook, 2nd ed. London \& Thousand Oaks, CA: Sage.

23. Jago R, Fox K, Page A et al. (2010) Parent and child physical activity and sedentary time: do active parents foster active children? BMC Public Health 10, 194.

24. Ventura A \& Birch L (2008) Does parenting affect children's eating and weight status? Int J Behav Nutr Phys Act 5, 15.

25. He M, Piche L, Beynon C et al. (2010) Screen-related sedentary behaviors: children's and parents' attitudes, motivations and practices. J Nutr Educ Behav 42, 17-25.

26. Hinkley T, Crawford D, Salmon J et al. (2008) Preschool children and physical activity: a review of correlates. $\mathrm{Am} \mathrm{J}$ Prev Med 34, 435-441.

27. Janssen I \& LeBlanc A (2011) Systematic review of the health benefits of physical activity and fitness in school-aged children and youth. Int J Behav Nutr Phys Act 7, 40.

28. van Duyn M \& Pivonka E (2000) Overview of the health benefits of fruit and vegetable consumption for the dietetics professional: selected literature. J Am Diet Assoc 100, 1511-1521.

29. Anderson J, Baird P, Davis R et al. (2009) Health benefits of dietary fiber. Nutr Rev 67, 188-205.

30. Jurkowski JM, Green Mills LL, Lawson HA et al. (2012) Engaging low-income parents in childhood obesity prevention from Start to Finish: a case study. J Community Health (Epublication ahead of print version).

31. Drewnowski A \& Specter S (2004) Poverty and obesity: the role of energy density and energy costs. Am J Clin Nutr 79, 6-16. 